\begin{tabular}{|c|c|c|c|c|}
\hline \multicolumn{5}{|c|}{ Richtwaarden voor referentiegebieden van bloed- en urinebestanddelen } \\
\hline bestanddeel & conditie & referentiegebied & eenheid & opmerking \\
\hline $\begin{array}{l}\text { ALAT } \\
\text { ASAT } \\
\text { LD } \\
\text { AF } \\
\gamma \mathrm{GT} \\
\text { pancreas- } \\
\quad \text { amylase } \\
\text { CK } \\
\text { CK-MB } \\
\text { troponine T } \\
\text { troponine I }\end{array}$ & $\begin{array}{l}\text { vrouw } \\
\text { man } \\
\text { vrouw } \\
\text { man }\end{array}$ & $\begin{aligned}<45 \\
<40 \\
<225 \\
<120 \\
<35 \\
<50 \\
\quad 13-53 \\
\\
<170 \\
<200 \\
<5 \\
<0,03 \\
-\end{aligned}$ & $\begin{array}{l}\mathrm{U} / 1 \\
\mathrm{U} / 1 \\
\mathrm{U} / 1 \\
\mathrm{U} / 1 \\
\mathrm{U} / \mathrm{l} \\
\mathrm{U} / 1 \\
\mathrm{U} / \mathrm{l} \\
\\
\mathrm{U} / \mathrm{l} \\
\mathrm{U} / \mathrm{l} \\
\mathrm{ng} / \mathrm{ml} \\
\mu \mathrm{g} / \mathrm{l} \\
-\end{array}$ & $\begin{array}{l}37^{\circ} \mathrm{C} \\
37^{\circ} \mathrm{C} \\
\text { IFCC-methode } \\
37^{\circ} \mathrm{C} \\
37^{\circ} \mathrm{C} \\
37^{\circ} \mathrm{C} \\
37^{\circ} \mathrm{C} \\
37^{\circ} \mathrm{C} \\
37^{\circ} \mathrm{C} \\
\\
\text { sterk methode } \\
\text { afhankelijk }\end{array}$ \\
\hline $\begin{array}{l}\text { TSH } \\
\text { FT4 (vrij T4) } \\
\text { cortisol }\end{array}$ & $\begin{array}{l}08.00 \text { uur } \\
16.00 \text { uur }\end{array}$ & $\begin{aligned} 0,4 & -4,0 \\
9 & -24 \\
150 & -700 \\
100 & -400\end{aligned}$ & $\begin{array}{l}\mathrm{mU} / \mathrm{l} \\
\mathrm{pmol} / \mathrm{l} \\
\mathrm{nmol} / \mathrm{l} \\
\mathrm{nmol} / \mathrm{l}\end{array}$ & \\
\hline $\begin{array}{l}\text { PSA } \\
\text { hCG (intact }+ \\
\quad \beta \text {-subunit) }\end{array}$ & $60-70$ jaar & $\begin{array}{l}<4,5 \\
<5\end{array}$ & $\mu \mathrm{g} / \mathrm{l}$ & \\
\hline $\begin{array}{l}\text { hemoglobine (Hb) } \\
\text { erytrocyten } \\
\text { hematocriet (Ht) } \\
\text { MCV } \\
\text { MCH } \\
\text { MCHC } \\
\text { reticulocyten } \\
\text { trombocyten } \\
\text { leukocyten } \\
\text { differentiatie: } \\
\text { neutrofiele } \\
\text { granulocyten } \\
\text { basofiele } \\
\text { granulocyten } \\
\text { eosinofiele } \\
\text { granulocyten } \\
\text { lymfocyten } \\
\text { monocyten } \\
\text { bezinking }\end{array}$ & $\begin{array}{l}\text { vrouw } \\
\text { man } \\
\text { vrouw } \\
\text { man } \\
\text { vrouw } \\
\text { man }\end{array}$ & \begin{tabular}{|c}
$7,5-10,0$ \\
$8,5-11,0$ \\
$3,8-5,5$ \\
$4,3-6,2$ \\
$0,36-0,47$ \\
$0,41-0,51$ \\
$80-100$ \\
$1,7-2,2$ \\
$19,3-22,7$ \\
$30-150$ \\
$150-400$ \\
$4,0-11,0$ \\
$2,0-7,5$ \\
\\
$<0,1$ \\
\\
$<0,5$ \\
$1,0-3,5$ \\
$0,3-1,0$ \\
$<20$ \\
$<15$
\end{tabular} & $\begin{array}{l}\mathrm{mmol} / \mathrm{l} \\
\mathrm{mmol} / \mathrm{l} \\
10^{12} / \mathrm{l} \\
10^{12} / \mathrm{l} \\
\mathrm{l} / \mathrm{l} \\
\mathrm{l} / \mathrm{l} \\
\mathrm{fl} \\
\mathrm{fmol} \\
\mathrm{mmol} / \mathrm{l} \\
10^{9} / \mathrm{l} \\
10^{9} / 1 \\
10^{9} / \mathrm{l} \\
10^{9} / 1 \\
10^{9} / 1 \\
10^{9} / 1 \\
10^{9} / 1 \\
10^{9} / 1 \\
\mathrm{~mm} / \mathrm{uur} \\
\mathrm{mm} / \text { uur }\end{array}$ & \\
\hline $\begin{array}{l}\text { APTT } \\
\text { PT } \\
\text { fibrinogeen } \\
\text { D-dimeer } \\
\text { antitrombine } \\
\text { INR (PT) }\end{array}$ & $\begin{array}{l}1 \mathrm{e} \text { intensiteit } \\
2 \mathrm{e} \text { intensiteit }\end{array}$ & $\begin{array}{c}<30-40 \\
<12-15 \\
2,0-4,0 \\
<0,5 \\
0,80-1,20 \\
2,5-3,5 \\
3,0-4,0\end{array}$ & $\begin{array}{l}\mathrm{s} \\
\mathrm{s} \\
\mathrm{g} / \mathrm{l} \\
\mathrm{mg} / \mathrm{l} \\
\mathrm{U} / \mathrm{ml}\end{array}$ & $\left\{\begin{array}{l}\text { indicatie van } \\
\text { bovengrens }\end{array}\right.$ \\
\hline
\end{tabular}




\section{CODEX MEDICUS}

SAMENGESTELD ONDER HOOFDREDACTIE VAN

$$
\text { prof.dr. R.O.B. Gans }
$$

GRONINGEN

$$
\text { prof.dr. P.E.Y. Van Schil }
$$

ANTWERPEN

$$
\text { prof.dr. J.P. Vandenbroucke }
$$

LEIDEN

$$
\text { prof.dr. C. van Weel }
$$

NIJMEGEN

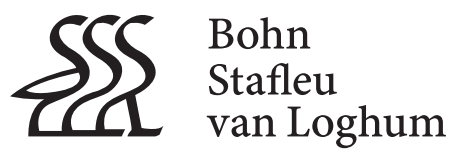


Eerste druk 1956

Tweede druk 1959

Derde druk 1964

Vierde druk 1968

Vijfde druk 1971

Zesde druk 1975

Zevende druk 1981

Achtste druk 1986

Negende druk 1991

Tiende druk 1996

Elfde druk 2001

Twaalfde druk 2005

Dertiende druk 2009, Reed Business Education, tweede en derde oplage 2010 en 2012

Veertiende (ongewijzigde) druk, Bohn Stafleu van Loghum, Houten 2016

ISBN 978-90-368-1628-1

ISBN 978-90-368-1629-8 (eBook)

DOI $10.1007 / 978-90-368-1629-8$

(C) 2016 Bohn Stafleu van Loghum, onderdeel van Springer Media BV

Alle rechten voorbehouden. Niets uit deze uitgave mag worden verveelvoudigd, opgeslagen in een geautomatiseerd gegevensbestand, of openbaar gemaakt, in enige vorm of op enige wijze, hetzij elektronisch, mechanisch, door fotokopieën of opnamen, hetzij op enige andere manier, zonder voorafgaande schriftelijke toestemming van de uitgever.

Voor zover het maken van kopieën uit deze uitgave is toegestaan op grond van artikel 16b Auteurswet jo het Besluit van 20 juni 1974, Stb. 351, zoals gewijzigd bij het Besluit van 23 augustus 1985, Stb. 471 en artikel 17 Auteurswet, dient men de daarvoor wettelijk verschuldigde vergoedingen te voldoen aan de Stichting Reprorecht (Postbus 3060, 2130 KB Hoofddorp). Voor het overnemen van (een) gedeelte(n) uit deze uitgave in bloemlezingen, readers en andere compilatiewerken (artikel 16 Auteurswet) dient men zich tot de uitgever te wenden.

Samensteller(s) en uitgever zijn zich volledig bewust van hun taak een betrouwbare uitgave te verzorgen. Niettemin kunnen zij geen aansprakelijkheid aanvaarden voor drukfouten en andere onjuistheden die eventueel in deze uitgave voorkomen.

NUR 871

www.codexmedicus.nl

Bohn Stafleu van Loghum

Het Spoor 2

Postbus 246

3990 GA Houten

www.bsl.nl 


\section{VOORWOORD}

Ruim 50 jaar geleden verscheen de eerste druk van de Codex Medicus. $\mathrm{Nu}$ ligt de nieuwe, dertiende druk voor u. Hoewel de opzet van de Codex gelijk is gebleven - een naslagwerk waarin u snel informatie vindt over medische begrippen die $\mathrm{u}$ in de eigen praktijkvoering niet dagelijks tegenkomt - is er in vergelijking met vorige drukken veel veranderd. Wegens emeritaat van een aantal redacteuren is er een vrijwel volledig nieuwe ploeg hoofdredacteuren en eindredacteuren aangetreden. Ook het auteursteam is ingrijpend vernieuwd. Dit heeft geleid tot een grondige bewerking van een aantal hoofdstukken. De Codex is daardoor optimaal geactualiseerd.

Graag willen wij de hoofdredacteuren en auteurs die niet meer aan deze Codex meewerken hierbij danken, in het bijzonder prof. L. Feenstra en prof. A.E. Meinders, alsmede Henk Deen, verantwoordelijke bureauredacteur bij Elsevier. Zij hebben een belangrijke bijdrage geleverd aan het succes van de Codex.

In deze nieuwe druk zijn drie nieuwe hoofdstukken toegevoegd: Jeugdgezondheidszorg, Gezondheidsrecht en Acute geneeskunde.

Hoewel de Codex zeer goed dienst doet als naslagwerk in boekvorm, wordt de informatie ook op elektronische manier aangeboden (zie www. codexmedicus.nl).

De Codex Medicus heeft een grote maatschappelijke betekenis, zoals onder meer blijkt uit de grote bereidheid van toonaangevende academici om als auteur de stand van kennis in hun vakgebied te presenteren. Aan deze dertiende druk heeft een recordaantal auteurs meegewerkt, namelijk 123. Het is bijzonder inspirerend om met zo'n grote groep medisch specialisten uit Nederland en Vlaanderen een naslagwerk actueel te houden. Veel auteurs zijn al jaren aan de Codex verbonden en blijven onverminderd enthousiast. Wij danken hen opnieuw voor hun bereidheid tot medewerking en samenwerking.

Wij zijn ervan overtuigd dat de Codex Medicus een nuttig en handig naslagwerk blijft voor huisartsen, specialisten, apothekers, tandartsen, paramedici, hoofdverpleegkundigen en vele andere professionals in de gezondheidszorg.

Graag nodigen wij u uit om uw kritische opmerkingen over de opzet en inhoud van de Codex Medicus door te geven. U kunt daartoe gebruikmaken van het volgende e-mailadres: redactie@codexmedicus.nl.

Oktober 2009

De Hoofdredactie,

R.O.B. GANS

P.E.Y. VAN SCHIL

J.P. VANDENBROUCKE

C. VAN Weel 
Voorwoord $\quad$ V

Inhoud, alfabetisch gerangschikt IX

$\begin{array}{ll}\text { Lijst van medewerkers } & X\end{array}$

Lijst van gebruikte afkortingen en tekens $\quad$ XVII

1. Bloedziekten, J.C. Kluin-Nelemans, A. Brand, F.J.M. van der Meer, W.E. Fibbe en R. Willemze

2. Endocrinologie, J.A. Romijn en J.W.A. Smit

3. Hart- en vaatziekten, F. Van de Werf, W.J.R. Desmet, M. Gewillig, H. Heidbuchel, M.C. Herregods, P.T. Sergeant en J. Vanhaecke

4. Aandoeningen van ademhalingstelsel, mediastinum en thoraxwand, M.L.A. Decramer, P.E.Y. Van Schil en J.F. Vansteenkiste

5. Leverziekten, ziekten van galwegen, pancreas en milt, W. Van Steenbergen, B. De Hemptinne en R.A. de Vries

6. Ziekten van buikholte en maag-darmkanaal, E.J. Kuipers, H.G. Gooszen en G. Hubens

7. Nierziekten, K.M.L. Leunissen, F.M. van der Sande en L. Verresen

8. Reumatische en systeemziekten, A.J.L. de Jong, J.M.W. Hazes en J.W.J. Bijlsma

9. Immuunziekten en allergie, H. Hooijkaas, H.A. Drexhage en J.L. Ceuppens

10. Infectieziekten, B.J. Kullberg, J.W.M. van der Meer en A. Warris

11. Oncologie, J.W.H. Leer en L.Y. Dirix

12. Geriatrie en gerontologie, J.P.J. Slaets en R.G.J. Westendorp

13. Ouderengeneeskunde, J.W.P.M. Konings, C.M.P.M. Hertogh en M.W. Ribbe

14. Tropische ziekten, P.A. Kager, A.M.L. van Gompel en B. Naafs 489

15. Intensivecaregeneeskunde, J.G. van der Hoeven en J.M.M. Verwiel

16. Ziekten van het zenuwstelsel, M. Vermeulen en P.R. Schuurman

17. Psychiatrie, W.J.G. Hoogendijk, H. van Engeland en N.M. Batelaan

18. Medische zorg voor verstandelijk gehandicapten, W.J. Braam en H.M. Evenhuis

19. Huisartsgeneeskunde, C. van Weel, J.A. Knottnerus, F.J.V.M. Buntinx en W.J.J. Assendelft

20. Kindergeneeskunde, R.J.B.J. Gemke

21. Jeugdgezondheidszorg, R.A. HiraSing, H. Talma en P. Van Royen

22. Vrouwenziekten, B.C.J.M. Fauser en T.J.M. Helmerhorst Page-Christiaensen en R. de Vlieger

24. Algemene chirurgie, R.H.G.G. Van Hee en W.J.H.J. Meijerink

25. Traumatologie, P.L.O. Broos en P. Patka

26. Urologie, J.J.J.M. Wyndaele en P. Hoebeke 
27. Orthopedische chirurgie, A.H.M. Taminiau en J. Somville

28. Plastische, reconstructieve en handchirurgie, C.M.A.M. van der Horst

29. Transplantatiegeneeskunde, R.J. Ploeg en W. van der Bij

31. Keel-, neus- en oorziekten, H.A.M. Marres

32. Mondziekten, kaak- en aangezichtschirurgie, K.G.H. van der Wal

33. Huid- en geslachtsziekten, C.A.F.M. Bruijnzeel-Koomen

34. Klinische genetica, cytogenetica en moleculaire genetica, E. De Baere, J.G. Leroy en A. De Paepe

35. Anesthesiologie en pijnbestrijding, L.H.D.J. Booij, W.W.A. Zuurmond en G.J. Scheffer

36. Radiologie, J.W.A.J. Reeders en H.R. Antonides

37. Nucleaire geneeskunde, B.L.F. van Eck-Smit

38. Revalidatie, H.J. Stam en G.G. Vanderstraeten

1275

39. Palliatieve zorg, C.C.D. van der Rijt, J.W.M. Krulder en H. van Veluw

40. Farmacotherapie en bijwerkingen van geneesmiddelen, C. Kramers, G.A. Rongen, P.A.G.M. de Smet en P. Smits

41. Vergiftigingen, J. Meulenbelt en I. de Vries

42. Voedings- en dieetleer, J.H.M. de Vries, E. Steenhagen en J.C. Seidell

43. Implantaten en biomaterialen, P. Patka
44. Bedrijfsgezondheidszorg, arbeidsomstandigheden en verzekeringsgeneeskunde, H.P. Goossens en T. Smid

45. Rampengeneeskunde, M.A.M. Debacker en J.J.L.M. Bierens

47. Klinische epidemiologie en biostatistiek, J.P. Vandenbroucke en J.G.P. Tijssen

48. Medische ethiek, E. van Leeuwen, P. Cras en W.M.P. Lemmens

49. Gezondheidsrecht, D.P. Engberts, H. Nys en Y.M. Koster-Reidsma

50. Acute geneeskunde, A.B. van Vugt 
4. Aandoeningen van ademhalingstelsel, mediastinum en thoraxwand

50. Acute geneeskunde

24. Algemene chirurgie

35. Anesthesiologie en pijnbestrijding

44. Bedrijfsgezondheidszorg, arbeidsomstandigheden en verzekeringsgeneeskunde

1. Bloedziekten

6. Buikholte en maag-darmkanaal, ziekten van

2. Endocrinologie

40. Farmacotherapie en bijwerkingen van geneesmiddelen

12. Geriatrie en gerontologie

49. Gezondheidsrecht

3. Hart- en vaatziekten

33. Huid- en geslachtsziekten

19. Huisartsgeneeskunde

46. Hyperbare en duikgeneeskunde

9. Immuunziekten en allergie

43. Implantaten en biomaterialen

10. Infectieziekten

15. Intensivecaregeneeskunde

21. Jeugdgezondheidszorg

31. Keel-, neus- en oorziekten

20. Kindergeneeskunde

1001

47. Klinische epidemiologie en biostatistiek

34. Klinische genetica, cytogenetica en moleculaire genetica

5. Leverziekten, ziekten van galwegen, pancreas en milt

48. Medische ethiek

18. Medische zorg voor verstandelijk gehandicapten

32. Mondziekten, kaak- en aangezichtschirurgie

7. Nierziekten

37. Nucleaire geneeskunde

11. Oncologie

30. Oogziekten 949

27. Orthopedische chirurgie $\quad 887$

13. Ouderengeneeskunde 475

39. Palliatieve zorg 1295

28. Plastische, reconstructieve en handchirurgie 911

17. Psychiatrie $\quad 579$

36. Radiologie 1219

45. Rampengeneeskunde 1427

8. Reumatische en systeemziekten 299

38. Revalidatie 1275

29. Transplantatiegeneeskunde 931

25. Traumatologie 845

14. Tropische ziekten 489

26. Urologie 865

41. Vergiftigingen 1327

23. Verloskunde $\quad 789$

42. Voedings- en dieetleer 1351

22. Vrouwenziekten 745

16. Zenuwstelsel, ziekten van het 531 


\section{LIJST VAN MEDEWERKERS}

DR. H.R. ANTONIDES, radioloog, Dr. J. Taamskliniek, Willemstad, Curacao, Nederlandse Antillen - medeauteur van hoofdstuk 36 Radiologie.

PROF.DR. W.J.J. ASSENDELFT, hoogleraar Huisartsgeneeskunde, Leids Universitair Medische Centrum - medeauteur van hoofdstuk 19 Huisartsgeneeskunde.

PROF.DR. E. DE BAERE, supervisor Laboratorium Moleculaire Genetica, Centrum Medische Genetica Gent. - medeauteur van hoofdstuk 34 Klinische genetica, cytogenetica en moleculaire genetica.

DRS. N.M. BATELAAN, psychiater GGZ inGeest en VU Medisch Centrum, Amsterdam - medeauteur van hoofdstuk 17 Psychiatrie.

PROF.DR. J.J.L.M. BIERENS, hoogleraar Urgentiegeneeskunde, VU Medisch Centrum, Amsterdam - medeauteur van hoofdstuk 45 Rampengeneeskunde.

DR. W. VAN DER BIJ, internist, Longtransplantatie-programma, Universitair Medisch Centrum Groningen - medeauteur van hoofdstuk 29 Transplantatiegeneeskunde.

PROF.DR. J.W.J. BIJLSMA, reumatoloog, hoofd afd. Reumatologie \& klinische Immunologie, Universitair Medisch Centrum Utrecht - medeauteur van hoofdstuk 8 Reumatische en systeemziekten.

DR. K.W.M. BLOEMENKAMP, gynaecoloog-perinatoloog, afd. Verloskunde, Leids Universitair Medisch Centrum - medeauteur van hoofdstuk 23 Verloskunde. PROF.DR. L.H.D.J. BOOIJ, hoogleraar Anesthesiologie, Universitair Medisch Centrum St Radboud, Nijmegen - medeauteur van hoofdstuk 35 Anesthesiologie en pijnbestrijding.

DRS. W.J. BRAAM, arts voor verstandelijk gehandicapten, 's Heeren Loo MiddenNederland, locatie Wekerom en Ziekenhuis Gelderse Vallei, Ede - medeauteur van hoofdstuk 18 Medische zorg voor verstandelijk gehandicapten.

PROF.DR. A. BRAND, internist-hematoloog, bijzonder hoogleraar transfusiegeneeskunde, Leids Universitair Medisch Centrum en manager afd. Onderzoek en Ontwikkeling, Sanquin Bloedbank regio Zuidwest - medeauteur van hoofdstuk 1 Bloedziekten.

PROF.DR. P.L.O. BROOS, gewoon hoogleraar Heelkunde, Katholieke Universiteit Leuven - medeauteur van hoofdstuk 25 Traumatologie.

PROF.DR. C.A.F.M. BRUIJNZEEL-KOOMEN, hoogleraar Dermatologie, Universitair Medisch Centrum Utrecht - auteur van hoofdstuk 33 Huid- en geslachts-ziekten.

PROF.DR. F.J.V.M. BUNTINX, hoogleraar, afd. Huisartsgeneeskunde Katholieke Universiteit Leuven en Universiteit Maastricht - medeauteur van hoofdstuk 19 Huisartsgeneeskunde.

PROF.DR. J.L. CEUPPENS, hoogleraar Allergologie en klinische Immunologie, Universitair Ziekenhuis Gasthuisberg, Katholieke Universiteit Leuven - medeauteur van hoofdstuk 9 Immuunziekten en allergie.

PROF.DR. P. CRAS, hoogleraar Neurologie, Universitair Ziekenhuis Antwerpen medeauteur van hoofdstuk 48 Medische ethiek.

PROF.DR. M.A.M. DEBACKER, gasthoogleraar Rampengeneeskunde, Vrije Universiteit Brussel - medeauteur van hoofdstuk 45 Rampengeneeskunde.

PROF.DR. M.L.A. DECRAMER, hoogleraar Longziekten en hoofd afd. Longziekten, Universiteit Ziekenhuis Gasthuisberg, Katholieke Universiteit Leuven medeauteur van hoofdstuk 4 Aandoeningen van ademhalingstelsel, mediastinum en thoraxwand. 
PROF.DR. W.J.R. DESMET, buitengewoon hoogleraar Cardiologie en kliniekhoofd Universiteit Ziekenhuis Gasthuisberg, Katholieke Universiteit Leuven medeauteur van hoofdstuk 3 Hart- en vaatziekten.

PROF.DR. R. DEVLIEGER, hoogleraar Verloskunde en adjunct-kliniekhoofd verloskunde-gynaecologie, Universiteit Ziekenhuis Gasthuisberg, Katholieke Universiteit Leuven - medeauteur van hoofdstuk 23 Verloskunde.

DR. L.Y. DIRIX, medisch-oncoloog, Algemeen Ziekenhuis Sint-Augustinus, GasthuisZusters Antwerpen - medeauteur van hoofdstuk 11 Oncologie.

PROF.DR. H.A. DREXHAGE, arts-medisch immunoloog en hoogleraar Autoimmuunziekten van endocriene organen, Erasmus MC, Rotterdam - medeauteur van hoofdstuk 9 Immuunziekten en allergie.

PROF.DR. B.L.F. VAN ECK-SMIT, hoogleraar Nucleaire geneeskunde, Academisch Medisch Centrum, Amsterdam - auteur van hoofdstuk 37 Nucleaire geneeskunde.

PROF. MR. DR. D.P. ENGBERTS, ethicus en jurist, hoofd sectie Ethiek en recht van de gezondheidszorg, Leids Universitair Medisch Centrum - medeauteur van hoofdstuk 49 Gezondheidsrecht.

PROF.DR. H. VAN ENGELAND, emeritus hoogleraar Kinder- en jeugdpsychiatrie, Universitair Medisch Centrum Utrecht - medeauteur van hoofdstuk 17 Psychiatrie.

PROF.DR. H.M. EVENHUIS, hoogleraar Geneeskunde voor verstandelijk gehandicapten, afd. Huisartsgeneeskunde, Erasmus MC, Rotterdam - medeauteur van hoofdstuk 18 Medische zorg voor verstandelijk gehandicapten.

PROF.DR. B.C.J.M. FAUSER, hoogleraar Voortplanting en Gynaecologie, Universitair Medisch Centrum Utrecht - medeauteur van hoofdstuk 22 Vrouwenziekten.

PROF.DR. W.E. FIBBE, hoogleraar Hematologie in het bijzonder stamcelbiologie, Leids Universitair Medisch Centrum - medeauteur van hoofdstuk 1 Bloedziekten.

PROF.DR. R.O.B. GANS, hoofdredacteur, hoogleraar interne geneeskunde, Universitair Medisch Centrum Groningen.

PROF.DR. R.J.B.J. GEMKE, hoogleraar Kindergeneeskunde, VU Medisch Centrum, Amsterdam - auteur van hoofdstuk 20 Kindergeneeskunde.

PROF.DR. M. GEWILLIG, hoogleraar en diensthoofd Kindercardiologie, Universiteit Ziekenhuis Gasthuisberg, Katholieke Universiteit Leuven - medeauteur van hoofdstuk 3 Hart- en vaatziekten.

PROF.DR. A.M.L. VAN GOMPEL, internist, hoofdarts Medische diensten en deeltijds hoofddocent Tropische ziekten, Instituut voor Tropische Geneeskunde Antwerpen en consulent Tropische Infectieziekten, Universitair Ziekenhuis Antwerpen - medeauteur van hoofdstuk 14 Tropische ziekten.

DRS. H.P. GOOSSENS, verzekeringsarts, UWV Leiden - medeauteur van hoofdstuk 44 Bedrijfsgezondheidszorg, arbeidsomstandigheden en verzekeringsgeneeskunde.

PROF.DR. H.G. GOOSZEN, hoogleraar Chirurgie en manager Onderzoek en Onderwijs, Divisie Heelkundige Specialismen, Universitair Medisch Centrum Utrecht - medeauteur van hoofdstuk 6 Ziekten van buikholte en maagdarmkanaal.

PROF.DR. J.M.W. HAZES, hoogleraar Reumatologie, Erasmus MC, Rotterdam medeauteur van hoofdstuk 8 Reumatische en systeemziekten.

PROF.DR. R.H.G.G. VAN HEE, emeritus hoofddocent Algemene heelkunde, Universiteit Antwerpen - medeauteur van hoofdstuk 24 Algemene chirurgie. PROF.DR. H. HEIDBUCHEL, kliniekhoofd en buitengewoon hoogleraar CardiologieElektrofysiologie, Universitair Ziekenhuis Gasthuisberg, Katholieke Universiteit, Leuven - medeauteur van hoofdstuk 3 Hart- en vaatziekten. PROF.DR. T.J.M. HELMERHORST, hoogleraar Verloskunde en vrouwenziekten, Erasmus MC, Rotterdam - medeauteur van hoofdstuk 22 Vrouwenziekten. 
PROF.DR. B. DE HEMPTINNE, hoogleraar Chirurgie, voorzitter Chirurgische Raad, Universitair Medisch Centrum, Gent - medeauteur van hoofdstuk 5 Leverziekten, ziekten van galwegen, pancreas en milt.

PROF.DR. M.C. HERREGODS, cardioloog, Universiteit Ziekenhuis Gasthuisberg, Katholieke Universiteit Leuven - medeauteur van hoofdstuk 3 Hart- en vaatziekten.

DR. C.M.P.M. HERTOGH, ouderenarts, VU Medisch Centrum, Amsterdam - medeauteur van hoofdstuk 13 Ouderengeneeskunde.

PROF.DR. R.A. HIRASING, hoogleraar Jeugdgezondheidszorg, VU Medisch Centrum, Amsterdam - medeauteur van hoofdstuk 21 Jeugdgezondheidszorg.

PROF.DR. P. HOEBEKE, diensthoofd Urologie, Kinderurologie en urogenitale reconstructie, Universitair Ziekenhuis Gent - medeauteur van hoofdstuk 26 Urologie. PROF.DR. J.G. VAN DER HOEVEN, hoogleraar Intensivecare, Universitair Medisch Centrum St Radboud, Nijmegen - medeauteur van hoofdstuk 15 Intensivecaregeneeskunde.

PROF.DR. W.J.G. HOOGENDIJK, hoogleraar Biologische psychiatrie, VU Medisch Centrum en GGZ inGeest, Amsterdam - medeauteur van hoofdstuk 17 Psychiatrie.

PROF.DR. H. HOOIJKAAS, hoogleraar Medische immunologie, Erasmus MC, Rotterdam - medeauteur van hoofdstuk 9 Immuunziekten en allergie.

PROF.DR. C.M.A.M. VAN DER HORST, hoogleraar Plastische reconstructieve en handchirurgie, Academisch Medisch Centrum, Amsterdam - auteur van hoofdstuk 28 Plastische, reconstructieve en handchirurgie.

PROF.DR. G. HUBENS, adjunct diensthoofd Abdominale heelkunde, Universitair Ziekenhuis Antwerpen - medeauteur van hoofdstuk 6 Ziekten van buikholte en maag-darmkanaal.

DR. R.A. VAN HULST, duikerarts-bedrijfsarts en directeur Maritiem Medisch Expertise Centrum/Duikmedisch Centrum, Koninklijke Marine, Den Helder auteur van hoofdstuk 46 Hyperbare en duikgeneeskunde.

DR. A.J.L. DE JONG, reumatoloog, Ziekenhuis Rijnstate Arnhem - medeauteur van hoofdstuk 8 Reumatische en systeemziekten.

PROF.DR. P.T.V.M. DE JONG, emeritus hoogleraar Epidemiologie van oogziekten, Erasmus MC, Rotterdam, oogarts-onderzoeker Nederlands Instituut voor Neurowetenschappen der KNAW, Amsterdam en oogarts Academisch Medisch Centrum, Amsterdam - medeauteur van hoofdstuk 30 Oogziekten. PROF.DR. P.A. KAGER, internist en emeritus hoogleraar Tropische geneeskunde Academisch Medisch Centrum, Amsterdam - medeauteur van hoofdstuk 14 Tropische ziekten.

PROF.DR. J.C. KLUIN-NELEMANS, hoofd afd. Hematologie, Universitair Medisch Centrum Groningen - medeauteur van hoofdstuk 1 Bloedziekten.

PROF.DR. J.A. KNOTTNERUS, hoogleraar Huisartsgeneeskunde, Universiteit Maastricht en voorzitter van de Gezondheidsraad, Den Haag - medeauteur van hoofdstuk 19 Huisartsgeneeskunde.

DR. J.W.P.M. KONINGS, ouderenarts, Zuwe Maria-Oord Zorgcentra, De Ronde Venen - medeauteur van hoofdstuk 13 Ouderengeneeskunde.

MR. Y.M. KOSTER-REIDSMA, jurist en onderzoeker-docent Gezondheidsrecht, sectie Ethiek en Recht van de Gezondheidszorg, Leids Universitair Medisch Centrum - medeauteur van hoofdstuk 49 Gezondheidsrecht.

DR. C. KRAMERS, internist klinisch farmacoloog, afd. Farmacologie-Toxicologie en Algemeen Inwendige Geneeskunde, Universitair Medisch Centrum St Radboud, Nijmegen en afd. Klinische Farmacie, Canisius Wilhelmina Ziekenhuis, Nijmegen - medeauteur van hoofdstuk 40 Farmacotherapie en bijwerkingen van geneesmiddelen.

DR. J.W.M. KRULDER, geriater, Vlietland Ziekenhuis, Schiedam - medeauteur van hoofdstuk 39 Palliatieve zorg. 
PROF.DR. E.J. KUIPERS, hoogleraar klinische gastroenterologie en hoofd afd. Interne Geneeskunde en Maag-, darm- en leverziekten, Erasmus MC, Rotterdam - medeauteur van hoofdstuk 6 Ziekten van buikholte en maagdarmkanaal.

PROF.DR. B.J. KULLBERG, internist-infectioloog en hoogleraar Infectieziekten, Universitair Medisch Centrum St Radboud, Nijmegen - medeauteur van hoofdstuk 10 Infectieziekten.

PROF.DR. J.W.H. LEER, hoogleraar Radiotherapie, Universitair Medisch Centrum St Radboud, Nijmegen - medeauteur van hoofdstuk 11 Oncologie.

PROF.DR. E. VAN LEEUWEN, hoogleraar Medische ethiek, Universitair Medisch Centrum St Radboud, Nijmegen - medeauteur van hoofdstuk 48 Medische ethiek.

PROF.DR. W.M.P. LEMMENS, hoofddocent Geschiedenis van de wijsbegeerte en ethiek, Universiteit Antwerpen - medeauteur van hoofdstuk 48 Medische ethiek.

PROF.DR. J.G. LEROY, emeritus gewoon hoogleraar Kindergeneeskunde, Universitair Ziekenhuis en Universiteit Gent - medeauteur van hoofdstuk 34 Klinische genetica, cytogenetica en moleculaire genetica.

PROF.DR. K.M.L. LEUNISSEN, internist-nefroloog afd. Interne geneeskunde Universiteit Maastricht - medeauteur van hoofdstuk 7 Nierziekten.

PROF.DR. G.P.M. LUYTEN, hoogleraar Oogheelkunde en hoofd afd. Oogheelkunde, Leids Universitair Medisch Centrum - medeauteur van hoofdstuk 30 Oogziekten.

PROF.DR. H.A.M. MARRES, hoogleraar Keel-, neus- en oorheelkunde, Universitair Medisch Centrum St Radboud, Nijmegen - auteur van hoofdstuk 31 Keel-, neus-en oorziekten.

DR. F.J.M. VAN DER MEER, internist, afd. Trombose en hemostase, Leids Universitair Medisch Centrum en directeur Stichting Trombosedienst Leiden en omstreken - medeauteur van hoofdstuk 1 Bloedziekten.

PROF.DR. J.W.M. VAN DER MEER, internist-infectioloog en hoofd afd. Algemeen interne geneeskunde, Universitair Medisch Centrum St Radboud, Nijmegen - medeauteur van hoofdstuk 10 Infectieziekten.

DR. W.J.H.J. MEIJERINK, minimaal invasieve en colorectale chirurgie, afd. Gastrointestinale heelkunde, VU Medisch Centrum, Amsterdam - medeauteur van hoofdstuk 24 Algemene chirurgie.

PROF.DR. J. MEULENBELT, internist - intensivist - toxicoloog, hoogleraar Klinische toxicologie, hoofd Nationaal Vergiftigingen Informatie Centrum, Rijksinstituut voor Volksgezondheid en Milieu, tevens Divisie Intensive Care Centrum, Universitair Medisch Centrum Utrecht en Instit - medeauteur van hoofdstuk 41 Vergiftigingen.

DR. B. NAAFS, dermatovenereoloog en staflid Leids Universitair Medisch Centrum; bestuurder Stichting voor Dermatologie en Venereologie Polderma, Emmeloord, gasthoogleraar aan het Regional Dermatology Training Centre (RDTC) Moshi, Tanzania en aan het Instituto Lauro de - medeauteur van hoofdstuk 14 Tropische ziekten.

PROF.DR. H. NYS, hoogleraar Medisch recht en directeur Centrum voor Biomedische Ethiek en Recht, Katholieke Universiteit Leuven - medeauteur van hoofdstuk 49 Gezondheidsrecht.

PROF.DR. A. DE PAEPE, hoogleraar Menselijke genetica en diensthoofd Centrum Medische Genetica Gent - medeauteur van hoofdstuk 34 Klinische genetica, cytogenetica en moleculaire genetica.

DR. G.C.M.L. PAGE-CHRISTIAENS, perinatoloog, Divisie Vrouw en Baby, Universitair Medisch Centrum Utrecht - medeauteur van hoofdstuk 23 Verloskunde.

PROF.DR. P. PATKA, traumachirurg, Erasmus MC, Rotterdam - auteur van hoofdstuk 43 Implantaten en biomaterialen en medeauteur van hoofdstuk 25 Traumatologie. 
PROF.DR. R.J. PLOEG, hoogleraar Heelkunde i.h.b. Transplantatiechirurgie, Universitair Medisch Centrum Groningen - medeauteur van hoofdstuk 29 Transplantatiegeneeskunde.

DR. J.W.A.J. REEDERS, Interventieradioloog, Sint Elisabeth Hospitaal, Willemstad, Nederlandse Antillen - medeauteur van hoofdstuk 36 Radiologie.

PROF.DR. M.W. RIBBE, hoogleraar ouderengeneeskunde, VU Medisch Centrum, Amsterdam - medeauteur van hoofdstuk 13 Ouderengeneeskunde.

DR. C.C.D. VAN DER RIJT, internist-oncoloog, Erasmus MC - Daniel den Hoed Oncologisch Centrum en voorzitter Kenniscentrum Palliatieve Zorg, Erasmus MC, Rotterdam - medeauteur van hoofdstuk 39 Palliatieve zorg. PROF.DR. J.A. ROMIJN, hoogleraar Interne geneeskunde, i.h.b. Endocrinologie, Leids Universitair Medisch Centrum - medeauteur van hoofdstuk 2 Endocrinologie. DR. G.A. RONGEN, internist, klinisch farmacoloog, vasculair geneeskundige, afd. Farmacologie-Toxicologie en Algemeen Interne Geneeskunde, Universitair Medisch Centrum St Radboud, Nijmegen - medeauteur van hoofdstuk 40 Farmacotherapie en bijwerkingen van geneesmiddelen.

PROF.DR. P. VAN ROYEN, huisarts, hoogleraar Huisartsgeneeskunde, Universiteit Antwerpen en docent in de interuniversitaire masteropleiding Jeugdgezondheidszorg - medeauteur van hoofdstuk 21 Jeugdgezondheidszorg.

DR. IR. J. RUINEMANS-KOERTS, klinisch chemicus, Klinisch Chemisch en Hematologisch Laboratorium, Alysis Zorggroep, Ziekenhuis Rijnstate, Arnhem auteur van de richtwaarden voor referentiegebieden van bloed- en urinebestand-delen.

DR. F.M. VAN DER SANDE, internist-nefroloog, Maastricht Universitair Medisch Centrum - medeauteur van hoofdstuk 7 Nierziekten.

PROF.DR. G.J. SCHEFFER, anesthesioloog-intensivist en hoogleraar Anesthesiologie Universitair Medisch Centrum St Radboud, Nijmegen - medeauteur van hoofdstuk 35 Anesthesiologie en pijnbestrijding.

PROF.DR. P.E.Y. VAN SCHIL, hoofdredacteur, diensthoofd en hoofddocent Thorax- en vaatheelkunde, Universiteit Antwerpen - medeauteur van hoofdstuk 4 Aandoeningen van ademhalingstelsel, mediastinum en thoraxwand.

DR. P.R. SCHUURMAN, neurochirurg, Academisch Medisch Centrum, Amsterdam medeauteur van hoofdstuk 16 Ziekten van het zenuwstelsel.

PROF.DR. J.C. SEIDELL, hoogleraar Voeding en gezondheid, Vrije Universiteit en VU Medisch Centrum, Amsterdam - medeauteur van hoofdstuk 42 Voedings-en dieetleer.

PROF.DR. P.T. SERGEANT, gewoon hoogleraar Heelkunde, Universitair Ziekenhuis Gasthuisberg, Katholieke Universiteit Leuven - medeauteur van hoofdstuk 3 Hart-en vaatziekten.

PROF.DR. J.P.J. SLAETS, hoogleraar Ouderengeneeskunde, Universitair Medisch Centrum Groningen - medeauteur van hoofdstuk 12 Geriatrie en gerontologie.

PROF.DR. P.A.G.M. DE SMET, apotheker-klinisch farmacoloog en bijzonder hoogleraar Kwaliteit van de farmaceutische patiëntenzorg, Universitair Medisch Centrum St Radboud, Nijmegen - medeauteur van hoofdstuk 40 Farmacotherapie en bijwerkingen van geneesmiddelen.

PROF.DR. IR. T. SMID, hoogleraar Arbeidsomstandigheden, VU Medisch Centrum, Amsterdam en manager Arbeidsveiligheid, KLM, Schiphol - medeauteur van hoofdstuk 44 Bedrijfsgezondheidszorg, arbeidsomstandigheden en verzekeringsgeneeskunde.

PROF.DR. J.W.A. SMIT, hoogleraar Interne geneeskunde in het bijzonder de klinische endocrinologie en diensthoofd afd. Endocrinologie, Leids Universitair Medisch Centrum - medeauteur van hoofdstuk 2 Endocrinologie.

PROF.DR. P. SMITS, internist-farmacoloog, afd. Farmacologie-toxicologie, Universitair Medisch Centrum St Radboud, Nijmegen - medeauteur van hoofdstuk 40 Farmacotherapie en bijwerkingen van geneesmiddelen. 
PROF.DR. J. SOMVILLE IR., hoofddocent en diensthoofd afd. Orthopedie en traumatologie, Universitair Ziekenhuis Antwerpen - medeauteur van hoofdstuk 27 Orthopedische chirurgie.

PROF.DR. H.J. STAM, hoogleraar Revalidatiegeneeskunde, Erasmus MC, Rotterdam medeauteur van hoofdstuk 38 Revalidatie.

PROF.DR. W. VAN STEENBERGEN, hoogleraar Leverziekten, Universitair Ziekenhuis Gasthuisberg, Katholieke Universiteit Leuven - medeauteur van hoofdstuk 5 Leverziekten, ziekten van galwegen, pancreas en milt.

E. STEenhagen, diëtist, Julius Centrum, afd. Diëtetiek en Voedingswetenschappen, Universitair Medisch Centrum Utrecht - medeauteur van hoofdstuk 42 Voedings- en dieetleer.

DRS. H. TALMA, sociaal-geneeskundige/jeugdarts, sectie Jeugdgezondheidszorg, afd. Sociale geneeeskunde, VU Medisch Centrum, Amsterdam, TNO Kwaliteit van Leven en GGD Hollands Noorden - medeauteur van hoofdstuk 21 Jeugdgezondheidszorg.

PROF.DR. A.H.M. TAMINIAU, hoogleraar Orthopaedische oncologie, Leidsch Universitair Medisch Centrum - medeauteur van hoofdstuk 27 Orthopedische chirurgie.

PROF.DR. J.G.P. TIJSSEN, hoogleraar Klinische epidemiologie en biostatistiek, Academisch Medisch Centrum, Amsterdam - medeauteur van hoofdstuk 47 Klinische epidemiologie en biostatistiek.

PROF.DR. J.P. VANDENBROUCKE, hoofdredacteur, hoogleraar Klinische epidemiologie, Leids Universitair Medisch Centrum en Akademiehoogleraar, Koninklijke Nederlandse Akademie van Wetenschappen - medeauteur van hoofdstuk 47 Klinische epidemiologie en biostatistiek.

PROF.DR. G.G. VANDERSTRAETEN, hoogleraar Fysische geneeskunde en revalidatie, Uni-versitair Ziekenhuis, Gent, België - medeauteur van hoofdstuk 38 Revalidatie.

PROF.DR. J. VANHAECKE, cardioloog, afd. Hartfalen-harttransplantatie, Universitair Ziekenhuis Gasthuisberg, Katholieke Universiteit Leuven - medeauteur van hoofdstuk 3 Hart-en vaatziekten.

PROF.DR. J.F. VANSTEENKISTE, longarts-oncoloog, Universitair Ziekenhuis Gasthuisberg, Katholieke Universiteit Leuven - medeauteur van hoofdstuk 4 Aandoeningen van ademhalingstelsel, mediastinum en thoraxwand.

H. VAN VELUW, verpleegkundig specialist en senior verpleegkundig consulent, Erasmus MC-Daniel den Hoed Oncologisch Centrum, Rotterdam - medeauteur van hoofdstuk 39 Palliatieve zorg.

PROF.DR. M. VERMEULEN, hoogleraar Neurologie, Academisch Medisch Centrum, Amsterdam - medeauteur van hoofdstuk 16 Ziekten van het zenuwstelsel.

PROF.DR. L. VERRESEN, internist-nefroloog, Ziekenhuis Oost-Limburg Genk en docent Pathofysiologie Universiteit Hasselt - medeauteur van hoofdstuk 7 Nierziekten.

DRS. J.M.M. VERWIEL, internist-intensivist, plaatsvervangend opleider afd. Intensivecare, Universitair Medisch Centrum St Radboud, Nijmegen medeauteur van hoofdstuk 15 Intensivecaregeneeskunde.

DR. R.A. DE VRIES, internist-MDL-arts afd. Inwendige geneeskunde, Universitair Medisch Centrum Groningen - medeauteur van hoofdstuk 5 Leverziekten, ziekten van galwegen, pancreas en milt.

DRS. I. DE VRIES, internist-toxicoloog, plaatsvervangend hoofd Nationaal Vergiftigingen Informatie Centrum, Rijksinstituut voor Volksgezondheid en Milieu en Divisie Intensive Care Centrum, Universitair Medisch Centrum Utrecht - medeauteur van hoofdstuk 41 Vergiftigingen.

DR. J.H.M. DE VRIES, voedingskundige en universitair docent Humane voeding, Wageningen Universiteit - medeauteur van hoofdstuk 42 Voedings- en dieetleer. 
PROF.DR. A.B. VAN VUGT, traumachirurg, Medisch Spectrum Twente, Enschede auteur van hoofdstuk 50 Acute geneeskunde.

PROF.DR. K.G.H. VAN DER WAL, hoogleraar Mondziekten, kaak- en aangezichtschirurgie, Erasmus MC, Rotterdam - auteur van hoofdstuk 32 Mondziekten, kaak- en aangezichtschirurgie.

DR. A. WARRIS, kinderarts-infectioloog/immunoloog, Universitair Medisch Centrum St Radboud, Nijmegen - medeauteur van hoofdstuk 10 Infectieziekten.

PROF.DR. C. VAN WEEL, hoofdredacteur, hoogleraar Huisartsgeneeskunde, afd. eerstelijnsgeneekunde, Universitair Medisch Centrum St Radboud, Nijmegen - medeauteur van hoofdstuk 19 Huisartsgeneeskunde.

PROF.DR. F. VAN DE WERF, hoogleraar en diensthoofd Cardiologie, Universiteit Ziekenhuis Gasthuisberg, Katholieke Universiteit Leuven - medeauteur van hoofdstuk 3 Hart- en vaatziekten.

PROF.DR. R.G.J. WESTENDORP, hoogleraar Interne geneeskunde, afd. Ouderengeneeskunde Leids Universitair Medisch Centrum en executive director, Leyden Academy on vitality and ageing - medeauteur van hoofdstuk 12 Geriatrie en gerontologie.

PROF.DR. R. WILLEMZE, hoogleraar Hematologie, Leids Universitair Medisch Centrum - medeauteur van hoofdstuk 1 Bloedziekten.

PROF.DR. J.J.J.M. WYNDAELE, gewoon hoogleraar Urologie, Universiteit Antwerpen medeauteur van hoofdstuk 26 Urologie.

PROF.DR. W.W.A. ZUURMOND, hoogleraar Anesthesiologie in het bijzonder pallatieve zorg en pijnbestrijding, VU Medisch Centrum en Hospice Kuria, Amsterdam - medeauteur van hoofdstuk 35 Anesthesiologie en pijnbestrijding. 


\section{LIJST VAN GEBRUIKTE AFKORTINGEN EN TEKENS}

\begin{tabular}{|c|c|}
\hline a. & arteria \\
\hline Aet. & etiologie \\
\hline Bijw. & bijwerkingen \\
\hline Compl. & complicaties \\
\hline Contra-ind. & contra-indicaties \\
\hline Diagn. & diagnose \\
\hline Diff. & differentiële diagnose \\
\hline Dos. & dosering \\
\hline Freq. & frequentie \\
\hline Fys.diagn. & fysische diagnostiek \\
\hline Fys.ond. & fysisch onderzoek \\
\hline Hist. & histologisch onderzoek \\
\hline i.e.z. & in engere zin \\
\hline Imm.fluoresc. & immunofluorescentie \\
\hline Ind. & Indicaties \\
\hline Interpr. & interpretatie \\
\hline i.v. & intraveneus \\
\hline Lab. & laboratoriumonderzoek \\
\hline Longf.ond. & longfunctie-onderzoek \\
\hline M. & morbus \\
\hline Path.-anat. & pathologisch-anatomisch onderzoek \\
\hline Pathofys. & pathofysiologie \\
\hline Prev. & preventie \\
\hline Prof. & profylaxe \\
\hline Progn. & prognose \\
\hline Rö. & röntgenonderzoek \\
\hline spp. & species, soorten \\
\hline Stralenbel. & stralenbelasting \\
\hline Sympt. & symptomen \\
\hline Techn. & techniek \\
\hline Th. & therapie \\
\hline Toep. & toepassingen \\
\hline v. & vena \\
\hline Verl. & verloop \\
\hline Voorber. & voorbereiding \\
\hline Voork. & vóorkomen \\
\hline
\end{tabular}

verwijsteken (naar een trefwoord binnen hetzelfde hoofdstuk) registered, aanduiding van een merkgeneesmiddel 